\title{
ON EXACT SOLUTIONS TO THE ZUFIRIA'S HIGHER-ORDER BOUSSINESQ TYPE EQUATION WITH GENERALIZED COEFFICIENTS
}

\author{
Cesar A. Gómez S. \\ Departamento de Matemáticas \\ Universidad Nacional de Colombia \\ Bogotá, Kra. 30 No. 45-03, COLOMBIA
}

\begin{abstract}
In this paper, we study the Zufiria's higher-order Boussinesq type equation with generalized coefficients from the point of view of its exact solutions. The improved tanhcoth method is used with the aim to obtain periodic and soliton solutions to the mentioned equation. The solutions obtained here are in a more general form from those obtained by authors of other works. We will see that the solutions for some particular cases can be derived from the obtained here.
\end{abstract}

AMS Subject Classification: $35 \mathrm{C} 05$

Key Words: improved tanh-coth method, Zufiria's equation, Boussinesq type equations, periodic and soliton solutions

\section{Introduction}

In the recent years, several equations with variable coefficients have appeared as important models associated to several physical problems. For instance, we can see [1], [2], [3] and references therein, where the authors have considered and studied equations such as

$$
\left\{\begin{array}{l}
u_{t}(x, t)+k_{1} u^{2}(x, t) u_{x}(x, t)+u_{x x x}(x, t)=0, \\
u_{t}(x, t)+k_{1} t^{n} u^{2}(x, t)+k_{2} t^{m} u_{x x x}(x, t)=0, \\
u_{t}(x, t)+k_{1} u^{n}(x, t) u_{x}(x, t)+u_{x x x}(x, t)=0 .
\end{array}\right.
$$

Received: $\quad$ March 4, 2016

Revised:

July 7, 2016

Published: September 27, 2016 (c) 2016 Academic Publications, Ltd. url: www.acadpubl.eu 
So that, the construction of exact solutions to nonlinear partial differential equations with variable coefficients is an important problem in mathematical physics today. In this paper, we consider the following Zufiria's higher-order Boussinesq type equation with generalized coefficients

$$
\left\{\begin{array}{l}
u_{t}+h_{x}+u u_{x}+\alpha(t) u_{x x x x}=0 \\
h_{t}+(h u)_{x}+\beta(t) u_{x x x}+\rho(t) u_{x x x x x}=0
\end{array}\right.
$$

being $u(x, t), h(x, t)$ the unknown functions and $\alpha(t), \beta(t), \rho(t)$ coefficients which can be depend of the variable $t$. Two particular cases of the previous system have been studied from the point of view of its exact solutions [4], [5]

$$
\left\{\begin{array}{l}
u_{t}+h_{x}+u u_{x}+\alpha u_{x x x x}=0, \\
h_{t}+(h u)_{x}+\frac{1}{3} u_{x x x}+\frac{2}{15} u_{x x x x x}=0,
\end{array}\right.
$$

and the so called variant Boussinesq equation [5]

$$
\left\{\begin{array}{l}
u_{t}+h_{x}+u u_{x}=0, \\
h_{t}+(h u)_{x}+u_{x x x}=0 .
\end{array}\right.
$$

Exact solutions to (3) have derived by the authors in [6] for the case $\alpha=0$, and by the authors in [4] for $\alpha=0, \alpha=1$ and $\alpha \neq 0$, being $\alpha$ a constant. Exact solutions to (4) have been derived by the authors in [5].

The Boussinesq type equations are used to modeling several problems in some branches of the physic and engineering, particulary in the study of water wave near to coastal areas. More applications can be see in [6] and references therein. The main objective of this paper, consist on the obtention of travelig wave solutions to (2) by using the improved tanh-coth method [7],[8]. Clearly, the solutions obtained here are in a more general form that those obtained in [4], [6] and, as particular case, solutions to (3) are derived changing the values of the coefficients. For (4), the same technique can be used.

\section{Using the Improved tanh-coth Method to Solve Equation (2)}

For solve equation (2) by means of the improved tanh-coth method, first we consider the transformation

$$
\left\{\begin{array}{l}
u(x, t)=v(\xi), \\
h(x, t)=w(\xi), \\
\xi=x+\lambda t+\xi_{0} .
\end{array}\right.
$$


Here, $\xi_{0}$ is an arbitrary constant. Substituting (5) into system (2) and after integrate each equation with respect to new variable $\xi$ we have the following system of ordinary differential equations

$$
\left\{\begin{array}{l}
\lambda v(\xi)+w(\xi)+\frac{1}{2} v(\xi)^{2}+\alpha(t) v^{\prime \prime \prime}(\xi)+K_{1}=0 \\
\lambda w(\xi)+v(\xi) w(\xi)+\beta(t) v^{\prime \prime}(\xi)+\rho(t) v^{\prime \prime \prime}(\xi)+K_{2}=0
\end{array}\right.
$$

where, $K_{1}$ and $K_{2}$ are the respective integration constants. From the second equation of (6) we have

$$
w(\xi)=\frac{1}{\lambda+v(\xi)}\left(-\beta(t) v^{\prime \prime}(\xi)-\rho(t) v^{\prime \prime \prime \prime}(\xi)-K_{2}\right) .
$$

Substituting this last expression into first equation in (6), the following ordinary differential equation is derived

$$
\begin{aligned}
\lambda^{2} v(\xi)+\frac{3 \lambda}{2} v^{2}(\xi)- & \beta(t) v^{\prime \prime}(\xi)-\rho(t) v^{\prime \prime \prime \prime}(\xi)-K_{2}+\frac{1}{2} v^{3}(\xi) \\
& +\lambda \alpha(t) v^{\prime \prime \prime}(\xi)+\alpha(t) v(\xi) v^{\prime \prime \prime}(\xi)+K_{1}(\lambda+v(\xi))=0
\end{aligned}
$$

The idea of the tanh-coth method consists on the search of solution to (8) in the form

$$
v(\xi)=\sum_{i=0}^{M} a_{i}(t) \phi(\xi)^{i}+\sum_{i=M+1}^{2 M} a_{i}(t) \phi(\xi)^{M-i},
$$

where $M$ is a positive integer determined by balancing and $\phi=\phi(\xi)$ satisfies the Riccati equation

$$
\phi^{\prime}(\xi)=r(t)+q(t) \phi(\xi)+p(t) \phi(\xi)^{2}
$$

The $a_{i}(t), i=1,2, \ldots, 2 M, r(t), p(t), q(t)$ are functions depending only of the variable $t$ to be determined later.

Substituting (9) into (8), and balancing $v^{\prime \prime \prime \prime}(\xi)$ with $v(\xi)^{3}$ is derived the following relation

$$
M+4=3 M
$$

therefore

$$
M=2 .
$$

So that (9) reduces to

$$
v(\xi)=a_{0}(t)+a_{1}(t) \phi(\xi)+a_{2}(t)(\phi(\xi))^{2}+a_{3}(t)(\phi(\xi))^{-1}+a_{4}(t)(\phi(\xi))^{-2} .
$$


Substituting (11) into (8), using (10) and equating to zero the coefficients of all powers of $\phi(\xi)$, we get a set of algebraic equations for the unknowns $a_{0}(t)$, $a_{1}(t), a_{2}(t), a_{3}(t), a_{4}(t), r(t), q(t), p(t)$. We solve the system with aid the Mathematica. The most general solution is listed below:

$$
\left\{\begin{array}{l}
a_{1}(t)=\frac{4 p(t) \rho(t)}{\alpha(t)}, \quad a_{3}(t)=\frac{\alpha(t)^{2} \beta(t)-4 \rho(t)^{2}}{4 \alpha(t)^{3} p(t)}, \\
r(t)=\frac{4 \rho(t)^{2}-\alpha(t)^{2} \beta(t)}{16 \alpha(t)^{2} p(t) \rho(t)}, \quad a_{0}(t)=-\lambda, \\
K_{1}=\frac{(-\lambda)^{2} \alpha(t)^{4}-4 \alpha(t)^{2} \beta(t) \rho(t)+16 \rho(t)^{3}}{2 \alpha(t)^{4}}, \quad K_{2}(t)=0, \\
a_{2}(t)=a_{4}(t)=q(t)=0 .
\end{array}\right.
$$

Now, its well known that the solution of (10) in the case $q(t)^{2}-4 p(t) q(t) \neq 0$ is given by (see [9]):

$$
\phi(\xi)=\frac{-\sqrt{q(t)^{2}-4 p(t) r(t)} \tanh \left[\frac{1}{2} \sqrt{q(t)^{2}-4 p(t) q(t)} \xi\right]-q(t)}{2 p(t)}
$$

Therefore, using the values given by (12), we have

$$
\phi(\xi)=-\frac{\sqrt{-\frac{4 \rho(t)^{2}-\alpha(t)^{2} \beta(t)}{\alpha(t)^{2} \rho(t)}} \tanh \left(\frac{1}{4} \sqrt{-\frac{4 \rho(t)^{2}-\alpha(t)^{2} \beta(t)}{\alpha(t)^{2} \rho(t)}} \xi\right)}{4 p(t)} .
$$

Finally, by (11), (7) and (5) we have that the solution of (2) corresponding to this values is given by

$$
\left\{\begin{array}{l}
u(x, t)=v(\xi)=a_{0}(t)+a_{1}(t) \phi(\xi)+a_{3}(t) \phi(\xi)^{-1}, \\
h(x, t)=w(\xi)=\frac{1}{\lambda+v(\xi)}\left(-\beta(t) v^{\prime \prime}(\xi)-\rho(t) v^{\prime \prime \prime \prime}(\xi)-K_{2}\right),
\end{array}\right.
$$

where $a_{0}(t), a_{1}(t), a_{3}(t)$ are given by $(12), \phi(\xi)$ given by $(14), p(t)$, arbitrary function wich can be depend on the the variable $t, \xi$ given by (5), where $\xi_{0}$ and $\lambda$ are arbitrary parameters.

Now, taking $\alpha(t)=\alpha, \beta(t)=\frac{1}{3}$ and $\rho(t)=\frac{2}{15}$ we have a solution to (3). Varying adequately the parameter $\xi_{0}$, periodic and soliton solutions are derived. 


\section{Conclusions}

We have derived exact solutions to the Zufira's higher-order Boussinesq type equations with variable coefficients. Two important particular cases have been considered, one of them called variant Boussinesq equation. Solutions for the first can be derived from the solutions obtained here by changing the parameters in an adequate form. Solutions for the variant Boussinesq equation can be derived applying the same technique used here.

\section{References}

[1] R.M. Miura, The Korteweg-de Vries equations and generalizations. A remarkable explicit nonlinear transformation, J. Math. Phys., 9 (1968), 1202-1204, doi: 10.1063/1.1664700.

[2] N. Nirmala, M.J. Vedan, B.V. Baby, Auto-Bäcklund transformation, Lax Pairs Painlevé property or a variable coefficient Korteweg-de Vries equation, J. Math. Phys., 27 (986), 2640-2646, doi: 10.1063/1.527282.

[3] Liu C. Yang, The applications of bifurvation method to a higher-order KdV equation, Math. Anal. Appl., 275 (2012), 1-12, doi: 10.1016/S0022-247X(02)00210-X.

[4] Liang Gao, Wen-Xiu Ma, Wei Xu, Traveling wave solutions to Zufiria's higherorder Boussinesq type equations, Nonlinear Analysis, 71 (2009), e711-e724, doi: 10.1016/j.na.2008.11.069.

[5] Kamruzzaman Khan, M. Ali Akbar, Traveling Wave Solutions of Some Coupled Nonlinear Evolution Equations, ISRN Mathematical Physic, 2013 (2013), Article ID 685736, 8 pages, doi: 10.1155/2013/685736.

[6] A.R. Seawady, O.H. El-Kalaawy, R.B. Aldenari, The applications of bifurvation method to a higher-order KdV equationWater wave solutions of Zufiria's higher-order Boussinesq type equations and its stability, Appl. Math and Comp., 280 (2016), 57-71, doi: 10.1016/j.amc.2016.01.014.

[7] C.A. Gomez S., An improved sub-equation method for solving nonlinear fractional equations, I.J.of pure and Appl. Mathematics, 101, No. 2 (2015), 133-140, doi: 10.12732/ijpam.v101i2.2.

[8] C.A. Gomez S., On a KdV equation with higher-order nonlinearity: Traveling wave solutions, Journal of Computational and Applied Mathematics, 235 (2011), 5330-5332, doi: $10.1016 /$ j.cam.2011.05.028.

[9] C.A. Gomez S and A. Salas, Special symmetries to standard Riccati equations and applications, Appl. Math, and Comp., 216, No. 10 (2010), 3089-3096, doi: 10.1016/j.amc.2010.04.039. 
